\title{
GROUPS OF ORDER 1
}

\section{ELVIRA STRASSER RAPAPORT}

I. Introduction. Let a group be given by generators, $x_{i}$, of the free group $F_{n}$ and defining relation, $R_{i}=1, i: 1, \cdots, n$, and denoted by

$$
G=\left(x_{1}, \cdots, x_{n} ; R_{1}, \cdots, R_{n}\right)=(x ; R) .
$$

One may wish to decide whether or not $G$ is trivial $(G=1)$. I shall discuss some properties of the trivial group and some decision techniques.

The symbols $G, R, R_{i}$ will refer to those given above.

II. Simple words. In a free group $F$ generated by the symbols $x_{1}, \cdots, x_{n}$, of rank $n$, a word (element) $w_{1}\left(x_{1}, \cdots, x_{n}\right)=w_{1}(x)$ is said to be simple (or a simple $x$-word) if there exist $n-1$ others, $w_{2}(x), \cdots, w_{n}(x)$, which together with $w_{1}(x)$ generate $F$. If $v_{1}(x)$, $\cdots, v_{n}(x)$ is another generating set of $x$-words, the set $v_{1}(x)$, $v_{2}(x), \cdots, v_{n}(x)$ may not generate $F$. That is, a collection of $n$ simple $x$-words may not be a generating set. I shall call the $x$-words $w_{1}, \cdots$, $w_{k}, k \leqq n$, of $F$ a set of simple words, or briefly a simple set, if they can be completed to (a "complete set of simple words") $w_{1}, \cdots, w_{n}$ that generate $F$.

The elements $R_{1}, \cdots, R_{n}$ of $F$ need not be simple or a simple set even if $G=1$. (Examples are given in $\S I I I$, below.) Whether or not they are can be determined and if they are then $x_{1}, \cdots, x_{n}$ are a simple set of $R$-words [6].

If they are not, the following rather obvious fact holds:

THEOREM 1. Suppose the elements $R_{1}, \cdots, R_{n}$ of the free group $F$ of rank $n$, generated by $x_{i}, \cdots, x_{n}$, have $t$ conjugates, $R_{1}^{\prime}, \cdots, R_{t}^{\prime}$, that can be written as a simple set (of g-words) in terms of the symbols $g_{i}=x_{j}^{k_{i}}, i: 1, \cdots, t, j: 1, \cdots, n$, and that those nonzero integers $k_{i} b e-$ longing to one subscript $j$ are relatively prime. Then the normal subgroup generated by $R_{1}, \cdots, R_{n}$ in $F$ is $F$ itself.

Proof. Since the $R_{i}^{\prime}$ are a complete set of simple $g$-words, they generate $g_{1}, \cdots, g_{t}$. As the latter, in their turn generate $x_{1}, \cdots, x_{n}$ the conclusion follows.

Note that the expressions so gotten for the $x_{j}$ in terms of the $R_{i}^{\prime}$ constitute a set of simple $R^{\prime}$-words. 1963.

Presented to the Society, September 10,1963; received by the editors June 21, 
The converse is not trivial and is contained in

Theorem 2. Suppose $G=\left(x_{1}, \cdots, x_{n} ; R_{1}, \cdots, R_{n}\right)$ is trivial, so that each $x_{i}$ is the product $f_{i}\left(R_{i j}, i: 1, \cdots, n, j: 1, \cdots, s\right)$ of $t$ conjugates $R_{i j}=z_{i j} R_{i} z_{i j}^{-1}$ of $R_{1}, \cdots, R_{n}$ in $F$, the free group generated by the $x_{i}$. Then $f_{1}, \cdots, f_{n}$ is a simple set of $R_{i j}$-words and possesses $a$ completion by $a$ set of $R_{i j}$-words, $f_{n+1}, \cdots, f_{t}$ which vanish qua $x$ words (i.e., when the symbols $R_{i j}$ are replaced by the $x$-words $R_{i j}(x)$ ).

Proof. If $\left(g_{1}, \cdots, g_{q} ; W_{1}, \cdots\right)$ is a free product

$$
G_{1} * G_{2} * \cdots * G_{k}
$$

of the groups $G_{i}$, then there is a set of simple $g$-words, $N\left(g_{1}\right), \cdots$, $N\left(g_{q}\right)$ each of which is contained in one or another of the factors $G_{i}$. If $W_{i}^{\prime}$ is the expression of $W_{i}$ written out in terms of the $N\left(g_{j}\right)$, then each $W_{i}^{\prime}$ belongs to some one of the factors (Grushko's theorem) [3].

Abbreviate $N\left(g_{i}\right)$ to $s_{i}$ and suppose $G$ above is isomorphic to the free group of rank $n, F_{n}$. Then one may take $G_{i}$ as free cyclic, say $G_{i}=F_{1}=F\left(y_{i}\right)$, with $y_{i}$ a certain $g$-word, $i: 1, \cdots, n$. It will follow that each $s_{i}$ is some power $y_{j}^{k_{i}}, j: 1, \cdots, n, i: 1, \cdots, q$.

Take a fixed $j$. If $s_{i}$ is $y_{j}$ or $y_{j}^{-1}$, then this $y_{j}$ is a simple $g$-word. Otherwise there must be several $s_{i}$ that are powers of this same $y_{j}$, say

$$
s_{1}=y_{1}^{k_{1}}, s_{2}=y_{1}^{k_{2}}, \cdots, s_{h}=y_{1}^{k_{h}}
$$

which together generate $y_{1}$. Being powers of $y_{1}$, these $s_{i}$ commute modulo the relations of $G$. Since, on the one hand $y_{1}$ is a power product $p\left(s_{1}, \cdots, s_{h}\right)$ of $s_{1}, \cdots, s_{h}$ in $G$, and on the other hand $s_{1}, \cdots, s_{h}$ are powers of $y_{1}$ in $G$, the nonzero integers among the $k_{i}$ are relatively prime. Therefore, there exists an element $C$ in the commutator subgroup $F_{h}^{\prime}$ of the free group $F_{h}$ generated by the $h$ symbols $s_{i}$ such that $p\left(s_{1}, \cdots, s_{h}\right) C$ is a simple $s$-word. In $G$, this word $C$ is the identity, so one may put $y_{1}=p C(s)$ ( $s$ denoting the collection of these $s_{i}$ ), whereby $y_{1}$ is expressed as a simple $s$-word. As any simple word in the free group $F_{h}$ has a completion, so does $p C(s)$. Set

$$
s_{1}^{*}=p C(s)
$$

(this is $y_{1}$ ) and $s_{2}{ }^{*}, \cdots, s_{h}^{*}$ a set that completes it in $F_{h}$. Then, in $G, s_{i}^{*}=y_{1}^{r_{i}}, i: 1, \cdots, h$, for some integers $r_{i}$. Furthermore, the words $s_{i}^{*} s_{1}^{*-r_{i}}, i: 2, \cdots, h$ also complete $s_{1}^{*}$ to a full set of free generators of $F_{h}$.

This procedure, carried out for each $j: 1, \cdots, n$, yields $n$ sets of 
simple $s$-words. As no $s_{i}$ appears in two of these sets, and as the total number of $s$-words gotten is $q$, they form a complete simple set of $s$-words in $F_{q}=F\left(s_{1}, \cdots, s_{q}\right)$.

As $s_{1}, \cdots, s_{q}$ are simple qua $g$-words, so is this new set. Note that it consists of the $n$ words $y_{1}(g), \cdots, y_{n}(g)$ that generate $G$ ( $G$ is now isomorphic to $\left.F_{n}\right)$ and $q-n$ words, say $y_{n+1}(g), \cdots, y_{q}(g)$, which are consequences of the defining relations of $G$.

Finally, to prove the theorem, view $g_{1}, \cdots, g_{q}$ as symbols for those conjugates $R_{1}^{\prime}, \cdots, R_{q}^{\prime}$ in $F=F\left(x_{1}, \cdots, x_{n}\right)$ of $R_{1}, \cdots, R_{n}$ of the theorems that appear in the expressions (assumed to exist) for $x_{1}, \cdots, x_{n}$ in the normal subgroup that $R_{1}, \cdots, R_{n}$ generate. Let $W_{1}, \cdots$ be those $g$-words in the free group $F_{q}=F\left(g_{1}, \cdots, g_{q}\right)$ which vanish identically when the $g_{i}$ are replaced by the $x$-words $R_{i}^{\prime}(x)$. Then the $y_{j}(g)$ are simple $R^{\prime}$-words and so generate the same subgroup as $R_{1}^{\prime}, \cdots, R_{q}^{\prime}$ do in $F_{q}=F\left(R_{1}^{\prime}, \cdots, R_{q}^{\prime}\right)$.

Now the symbol $R_{i}^{\prime}$ stands for an $x$-word in the originally defined group $G$, so $y_{j}\left(R^{\prime}(x)\right)$ is an $x$-word there, say $z_{j}(x)$. The $z_{j}(x)$ generate a subgroup of $F=F\left(x_{1}, \cdots, x_{n}\right)$. As the $R_{l}^{\prime}(x)$ generate $F$, the complete set of simple $R^{\prime}$-words $y_{j}\left(R^{\prime}(x)\right)=z_{j}(x)$ also do. As $y_{n+1}\left(R^{\prime}(x)\right)$, $\cdots, y_{4}\left(R^{\prime}(x)\right)$ vanish identically qua $x$-words, we have that $z_{n+1}(x)$ $=z_{n+2}(x)=\cdots=z_{q}(x)=1$, identically in $F\left(x_{1}, \cdots, x_{n}\right)$. Hence $z_{1}(x), \cdots, z_{n}(x)$ generate this free group, with each $z_{i}(x)$ giving rise to a free factor.

The free factors $G_{i}=F\left(y_{i}\right), i: 1, \cdots, n$, posited above can be chosen in advance from among all possible factorizations of $G$ into $n$ free factors. So, if $G$ is $F\left(x_{1}, \cdots, x_{n}\right)$, the free cyclic factors $F\left(x_{i}\right)$ may be chosen as the $G_{i}$. Then $F\left(x_{i}\right)=F\left(z_{i}(x)\right)$.

Because the only elements in $F\left(x_{i}\right)$ that generate it are $x_{i}$ and its inverse, the element $z_{i}(x)$ must be one of these.

This concludes the proof of Theorem 2 .

REMARK 1. Nielsen's method can also be used to prove this. The method is described in [1].

REMARK 2. One reason why Theorem 2 fails to give an effective procedure to decide whether $G$ is trivial is exemplified in (b) of §III. The finite process that works in the first group mentioned under (a) of \$III yields only a proper subgroup, $H$, of $F$, while the normal closure of $H$ is, of course, $F$. This is verified easily by Nielsen's method referred to above. It follows that at least one conjugate $z R_{i} z^{-1}$, of some $R_{i}$, is needed to generate $F$ in the sense of Theorem 2 that has longer $x$-length (the number of $x$-symbols in a word) than the $x$-length of $R_{i}$; there is no general method to find this $z R_{i} z^{-1}$. 
Let $s_{i}$ again stand for a simple $x$-word in $F=F_{n}$, with $F=F\left(x_{1}, \cdots, x_{n}\right)=F\left(s_{1}, \cdots, s_{n}\right), \quad s_{i}=s_{i}(x)=s_{i}\left(x_{1}, \cdots, x_{n}\right)$.

All the groups of $\S$ III have the property that $R_{i}$ vanishes modulo $s_{i}$ for some complete set $s_{1}, \cdots, s_{n}$, of simple words. In all but the first example $s_{1}=a, s_{2}=b, s_{3}=c$. Replacing $R_{i}$ by $s_{i}$ in groups with this property yields a factor-group. It is an interesting but difficult problem, posed by Magnus, to decide whether some $R_{i}$ may always be replaced by an $s_{i}$ whenever $G=1$.

The statement that if $G=\left(x_{1}, \cdots, x_{n} ; R_{1}, \cdots, R_{n}\right)=1$ then $R_{1}, \cdots, R_{k}$ can be replaced by $x_{1}, \cdots, x_{k}$ for every $k: 1, \cdots, n$ is obviously equivalent to either of the following two:

(1) Modulo $R_{k+1}, \cdots, R_{n}$, the words $R_{1}, \cdots, R_{k}$ are consequences of $x_{1}, \cdots, x_{k}, k: 1, \cdots, n$. In symbols: $R_{i}=V_{i} W_{i}$, with $V_{i}$ vanishing modulo $x_{1}, \cdots, x_{k}$, and $W_{i}$ vanishing modulo $R_{k+1}$, $\cdots, R_{n} ; i: 1, \cdots, k$.

(2) $R_{k+1}, \cdots, R_{n}$ generate $x_{k+1}, \cdots, x_{n}$ modulo $x_{1}, \cdots, x_{k}$. In symbols: $x_{i}=V_{i} W_{i}$, with $V_{i}$ vanishing modulo $R_{k+1}, \cdots, R_{n}$, and $W_{i}$ vanishing modulo $x_{1}, \cdots, x_{k} ; i: k+1, \cdots, n$.

Of course, neither (1) nor (2) suffices to make $G$ trivial. Are they necessary? I have no counterexample. However, when $n=2$, these conditions are always met (up to an automorphism of $F=F_{2}$ ). This follows from two observations: in the free group $F_{2}$, generated by $x_{1}$ and $x_{2}$, any element of the commutator subgroup vanishes modulo either $x_{i}$; if the normal subgroup $R_{1}$ and $R_{2}$ generate in $F_{2}$ is $F_{2}$ itself, then modulo commutators, $R_{i}=s_{i}$ for some pair of generators $s_{i}=s_{i}(x), i: 1,2$. Thus one may write $F_{2}$ and the $R_{i}$ in terms of the $s_{i}$, so that

$$
G=\left(s_{1}, s_{2} ; s_{1} C_{1}, s_{2} C_{2}\right)
$$

with the $C_{i}$ elements of the commutator subgroup of $F_{2}$. As $s_{i} C_{i}$ vanishes modulo $s_{i}$, the conclusion follows.

This is a special case of restricting the integer $k$ to the values $n-1$ and $n$, with $n$ arbitrary. Here too, the conditions (1) and (2) are always met (up to an automorphism of $F_{n}$ ). The reasons are entirely analogous to the case $n=2$.

III. Examples. (a) If $R_{1}$ and $R_{2}$ are words in the free group $F$ generated by $a$ and $b$, form their cyclically reduced conjugates $T_{1}, \cdots, T_{k}$. (For example, the cyclically reduced conjugates of the word $a b b$ are $a b b, b a b, b b a$.) Add to this set all products $T_{i} T_{j}^{ \pm 1}$ that are shorter than 
one of the words $T_{i}, T_{j}$. Repeat the procedure until nothing new turns up.

For

$$
R_{1}=a^{2} b^{3}, \quad R_{2}=a^{3} b^{4}
$$

this yields a set of $a, b$-words containing the words $a$ and $b$; therefore

$$
G=\left(a, b ; a^{2} b^{3}, a^{3} b^{4}\right)=1 .
$$

This result also takes care of the group

$$
\begin{aligned}
G^{*} & =\left(a, b ; a b^{-2} a b a^{-1} b a^{-1} b, a b^{-1}\left(b a^{-1} b a^{-1} b\right)^{-1} a b^{-2} a\left(b a^{-1} b a^{-1} b\right)^{2}\right) \\
& =\left(a, b ; a C_{1}, b C_{2}\right),
\end{aligned}
$$

$C_{1}, C_{2}$ elements of the commutator subgroup of $F(a, b)$. When one applies automorphisms of the free group on $a$ and $b$ to the words $a C_{1}$ and $b C_{2}$ to reduce their $a, b$-length (a finite procedure [6]), one arrives at $a^{2} b^{3}$ and $a^{3} b^{4}$. It follows that $G^{*}$ is also trivial.

(b) The group

$$
\left(a, b, c ; b a b^{-1} a^{-2}, c b c^{-1} b^{-2}, a c a^{-1} c^{-2}\right)=\left(a, b, c ; R_{1}, R_{2}, R_{3}\right)
$$

is trivial [5]. The method just used for $G^{*}$ fails to discover this.

This group is a special case of

$$
\left(a, b, c ; b a b^{-1} a^{-t-1}, c b c^{-1} b^{-t-1}, a c a^{-1} c^{-t-1}\right)
$$

with $t$ a fixed integer. When $t$ is greater than 1 , this group is not trivial [4].

(c) Is the group

$$
G=\left(a, b, c ; a^{-1} b^{2} a b^{-3}, b^{-1} c^{2} b c^{-8}, c^{-1} a^{2} c a^{-3}\right)=\left(a, b, c ; R_{1}, R_{2}, R_{3}\right)
$$

trivial?

Observe that $R_{1}$ can be written as

$$
u_{1} v_{1}^{2} u_{1} v_{1}^{-1} u_{1}^{-1} v_{1}^{-1}
$$

with

$$
\begin{aligned}
& u_{1}=\left(a^{-1} b a b^{-1}\right)^{2} b^{-1}, \\
& v_{1}=b^{2} a^{-1} b^{-1} a .
\end{aligned}
$$

The corresponding statement for $R_{2}$ and $R_{3}$ gives three words $u_{1}(a, b)$, $u_{2}(b, c), u_{3}(a, c)$; the $R_{i}$ are consequences of these words. Then, factoring out the $u_{i}$ from $G$ gives

$$
H=G / u_{1}, u_{2}, u_{3}=\left(a, b, c ; u_{1}, u_{2}, u_{3}\right) .
$$


This group $H$ satisfies the conditions of Greendlinger's theorem [2], which means that every consequence of $u_{1}, u_{2}, u_{3}$ contains at least five symbols. It follows that $G$ cannot be trivial.

One can investigate the more general group

$$
G_{k}=\left(A, B, C ; A^{-k} B^{2} A^{k} B^{-3}, B^{-k} C^{2} B^{k} C^{-3}, C^{-k} A^{2} C^{k} A^{-3}\right)
$$

along similar lines. For example, by noting that the mapping

$$
\begin{aligned}
& B \rightarrow a^{-1} b a b^{-1} \\
& C \rightarrow b^{-1} c b c^{-1} \\
& A \rightarrow c^{-1} a c a^{-1}
\end{aligned}
$$

is an isomorphism between $G_{2}$ and $H$, one finds that

$$
G_{2}=\left(a, b, c ; a^{-2} b^{2} a^{2} b^{-3}, b^{-2} c^{2} b^{2} c^{-2}, c^{-2} a^{2} c^{2} a^{-3}\right)
$$

is not trivial.

\section{BIBLIOGRAPHY}

1. H. Federer and B. Jonsson, Some properties of free groups, Trans. Amer. Math. Soc. 68 (1950), 1-27.

2. M. Greendlinger, Dehn's algorithm for the word problem, Comm. Pure Appl. Math. 13 (1960), 67-83.

3. A. G. Kurosh, The theory of groups, Chelsea, New York, 1956.

4. Jens Mennicke, Einige endliche Gruppen mit drei Erzeugenden und drei Relationen, Arch. Math. 10 (1959), 409-418.

5. B. H. Neumann, On some finite groups with trivial multiplicator, Publ. Math. Debrecen 4 (1956), 190-194.

6. E. S. Rapaport, On free groups and their automorphisms, Acta Math. 99 (1958), 139-163.

Polytechnic Institute of Brooklyn 